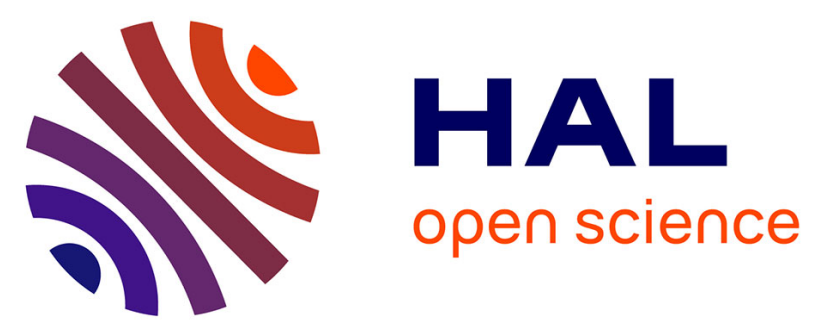

\title{
Analysis of magnetization loops of electrospun nonwoven superconducting fabrics
}

Xian Lin Zeng, Thomas Karwoth, Michael Rudolf Koblischka, Uwe Hartmann, Denis Gokhfeld, Crosby S Chang, Thomas Hauet

\section{To cite this version:}

Xian Lin Zeng, Thomas Karwoth, Michael Rudolf Koblischka, Uwe Hartmann, Denis Gokhfeld, et al.. Analysis of magnetization loops of electrospun nonwoven superconducting fabrics. Physical Review Materials, 2017, 1 (4), pp.044802. 10.1103/PhysRevMaterials.1.044802 hal-01710775

\section{HAL Id: hal-01710775 \\ https://hal.science/hal-01710775}

Submitted on 16 Feb 2018

HAL is a multi-disciplinary open access archive for the deposit and dissemination of scientific research documents, whether they are published or not. The documents may come from teaching and research institutions in France or abroad, or from public or private research centers.
L'archive ouverte pluridisciplinaire HAL, est destinée au dépôt et à la diffusion de documents scientifiques de niveau recherche, publiés ou non, émanant des établissements d'enseignement et de recherche français ou étrangers, des laboratoires publics ou privés. 


\title{
Analysis of magnetization loops of electrospun nonwoven superconducting fabrics
}

\author{
Xian Lin Zeng, Thomas Karwoth, Michael R. Koblischka, ${ }^{*}$ and Uwe Hartmann \\ Institute of Experimental Physics, Saarland University, Campus C 6 3, 66123 Saarbrücken, Germany \\ Denis Gokhfeld \\ Kirensky Institute of Physics, Federal Research Center KSC SB RAS, Akademgorodok 50/38, Krasnoyarsk 660036, Russia \\ Crosby Chang and Thomas Hauet \\ Institut Jean Lamour, UMR CNRS-Université de Lorraine, 54506 Vandoevre-lès-Nancy, France
}

(Received 16 February 2017; published 8 September 2017)

\begin{abstract}
Networks of superconducting $\mathrm{Bi}_{2} \mathrm{Sr}_{2} \mathrm{CaCu}_{2} \mathrm{O}_{8}$ (Bi-2212) nanowires were fabricated by the electrospinning technique. The nanowires have a diameter of the order of 150-200 nm and lengths up to the micrometer range and form a nonwoven, fabric-like network with numerous interconnections enabling a current flow between the nanowires. The porosity of this nanowire network is 0.9928 . Therefore, this material represents a novel class of ultraporous high-temperature superconductors. The magnetizations of the nanowire networks $[M(T)$ and $M(H)]$ were recorded by SQUID magnetometry. The magnetic properties were analyzed using the extended critical state model (ECSM). It is supposed that the averaged diameter of the nanowires rules the magnetic field dependence of the critical current density of the nanowire network. Single nanowires have remarkably high values of the critical current density of $1.04 \times 10^{7} \mathrm{~A} / \mathrm{cm}^{2}$ at $5 \mathrm{~K}$. The macroscopic critical current density less than $\sim 0.05 \mathrm{~A} / \mathrm{cm}^{2}$ at $5 \mathrm{~K}$ is fine for this lightweight material. Using ECSM, several important magnetic parameters could be determined including the penetration field $H_{p}$, the irreversibility fields $H_{\text {irr }}$, the upper critical field $H_{c 2}$, and the flux pinning forces. Applications for this material class may be found in the direction of sensors, thin shielding layers, or nanoporous bulks.
\end{abstract}

DOI: 10.1103/PhysRevMaterials.1.044802

\section{INTRODUCTION}

The ceramic nature of the high-temperature superconductors (HTSc) has laregly hindered many proposed applications of superconductivity. Therefore, different and better adapted fabrication routes for HTSc are required for various future applications. For superconducting tapes, CVD-based methods were developed for a second generation of tapes [1], but in other areas of superconductivity such as bulk samples and thin films, there has only been a little progress [2,3]. To fabricate fault-current limiters or effective magnetic shieldings, a different type of material is needed as it is desirable to combine the possibility of shaping with high critical current densities and light weight.

Superconducting fabrics are a novel class of superconducting materials formed by HTSc which could fulfill the needs mentioned above. In previous work published in the literature, which meets the requirement of shaping and light weight, woven $\mathrm{Y}_{2} \mathrm{O}_{3}$ cloths were employed as a base material, and the superconductor was formed by an infiltration growth process [4]. In the last years, this process has also been beneficial for bulk superconductors [5,6]. Superconducting fabrics of about $100 \mu \mathrm{m}$ thickness could be prepared using $\mathrm{YBa}_{2} \mathrm{Cu}_{3} \mathrm{O}_{x}$ (YBCO) as superconducting material [7,8]. An orientation of the grain growth was achieved by using seed crystals like for bulk superconductors [9]. As a result, such superconducting fabrics could carry currents of $5.5 \times 10^{4} \mathrm{~A} / \mathrm{cm}^{2}$, determined by means of electric measurements [10].

*Corresponding author: m.koblischka@mx.uni-saarland.de
The recent developments in nanotechnology enable the fabrication of nonwoven nanowire networks of ceramic materials by means of electrospinning [11-13]. Very recently, also superconducting nanowires and their networks were fabricated by this method [14-19]. The nanowires prepared by the electrospinning technique are polycrystalline, and these nanowires exhibit long lengths of up to $100 \mu \mathrm{m}$ with a diameter of the order of 100-200 $\mathrm{nm}$. The networks are formed directly in the electrospinning process, and the individual nanowires have multiple interconnections with each other. In a second step, the nanowire networks are heat treated to remove the organic compounds and to form the superconducting phase. During this processing step, the interconnections are converted into proper contact elements, which enable the flow of transport currents through the entire sample. In this way, a fabric-like structure results which is extremely light due to the high porosity; the resulting density of the material is only about $0.05 \mathrm{~g} / \mathrm{cm}^{3}$.

Due to the increased complexity of the resulting current flow, the analysis of the magnetization data measured by magnetometry is a nontrivial task, as the overall data consist of several contributions, i.e., currents within the superconducting grains, through the grain boundaries, and through the various interconnections between the individual nanowires. The application of the Bean model [20], which represents the simplest formula for critical current estimation, is questionable for superconducting samples with grains smaller than the magnetic penetration depth $\lambda$ and the coherence length $\xi$. A more realistic approximation than the Bean model is given by the extended critical state model (ECSM) [21-24] that accounts for the specific reversible magnetization of a surface layer of a superconducting sample. Thus, ECSM can fit the 
resulting asymmetric $M(H)$ loops of the nanowire network samples. In this way, it should be possible to obtain a better understanding of the current flow in the nanowire fabrics, enabling possible applications of this new type of material.

Therefore, the aim of this study is to carefully analyze the magnetic data of the nanowire network samples using both the Bean approach and the ECSM model in order to elucidate the important features of the current flow in these samples.

This paper is organized as follows: In Sec. II, the experimental procedures are outlined. Section III presents the results concerning the microstructure and the magnetic measurements of the $\mathrm{Bi}_{2} \mathrm{Sr}_{2} \mathrm{CaCu}_{2} \mathrm{O}_{x}$ (Bi-2212) nanowire network samples. Furthermore, the magnetic field dependencies of the critical current densities and the pinning forces resulting from the application of the Bean model and ECSM are presented. Section IV contains a discussion of the results obtained and some possible applications of this material class are outlined. Finally, in Sec. V some conclusions are drawn.

\section{EXPERIMENTAL PROCEDURE}

The Bi-2212 nanowire networks were fabricated using the electrospinning technique from an aqueous solution of polyvinylpyrrolidone (PVP, $M_{W} 1300$ 000), bismuth(III) acetate, strontium acetate hydrate, calcium acetate hydrate, and copper(II) acetate monohydrate and followed by calcination treatment of the electrospun polymer/inorganic composite fibers including one oxygenation step in pure $\mathrm{O}_{2}$. The chemicals were commercially available (Alfa Aesar GmbH and Co KG; purity above 99.99\%) and used as received without further purification. X-ray analysis confirmed that the samples are pure Bi-2212 phase with some residing carbon. Further details of the nanowire fabrication procedure are given elsewhere [15,16] and in the Supplemental Material [55]. Handling of the as-prepared flakes of the nanowire networks requires careful treatment, but is possible using tweezers and plasticine.

SEM imaging was performed using a Hitachi S800 scanning electron microscope operating at $10 \mathrm{kV}$. The TEM analysis was performed by a JEOL JSM-7000 F transmission electron microscope operating at $200 \mathrm{kV}$ with $\mathrm{a} \mathrm{LaB}_{6}$ cathode. The nanowire samples were mounted via solution on a standard TEM copper grid covered with a carbon film with a thickness less than $10 \mathrm{~nm}$. The spatial resolution of the SEM is about $1.2 \mathrm{~nm}$; the one of the TEM $0.14 \mathrm{~nm}$. In EDX mode, the beam diameter can be reduced to about $1.5 \mathrm{~nm}$, and the resolution is about $1 \%$.

The magnetization of the nanowire networks was measured using a SQUID magnetometer (Quantum Design MPMS3) with $\pm 7 \mathrm{~T}$ magnetic field applied perpendicular to the sample surface.

\section{RESULTS}

\section{A. Microstructure}

The microstructures of the $\mathrm{Bi}-2212$ nanowires are presented in Figs. 1(a) and 1(b) and in the Supplemental Material [55]. Figure 1(a) presents an SEM image of the nanowire network. The nanowire fabric has an open porous structure with numerous interconnections between the long individual nanowires.



(b)



FIG. 1. (a) SEM image (magnification $5000 \times$ ) of the nanowire network after the final heat treatment. (b) TEM bright-field image, revealing the polycrystalline character of the individual nanowires and details of the elongated $\mathrm{Bi}-2212$ grains. The red lines highlight the grain boundaries within the nanowires, while the blue circle indicates an interconnection between nanowires.

In Fig. 1(b), a TEM image of several individual nanowires is shown. The nanowires consist of several elongated grains which is typical for the growth of the Bi-2212 phase. The red lines highlight some grain boundaries within the nanowires, demonstrating the intergrain weak links between the grains. There is, however, no preferred orientation for the grains, so many high-angle grain boundaries result. The interconnections between the nanowires may extend over some grains as revealed by the darker regions, as shown in the blue circled region.

The density of our nanowire networks is found to be equal to $0.0459 \mathrm{~g} / \mathrm{cm}^{3}$, which is considerably lower than the theoretical density of bulk Bi-2212 $\left(6.4 \mathrm{~g} / \mathrm{cm}^{3}\right)$ [25]. As a result, the nanowire network samples in the as-prepared state are extremely brittle. 


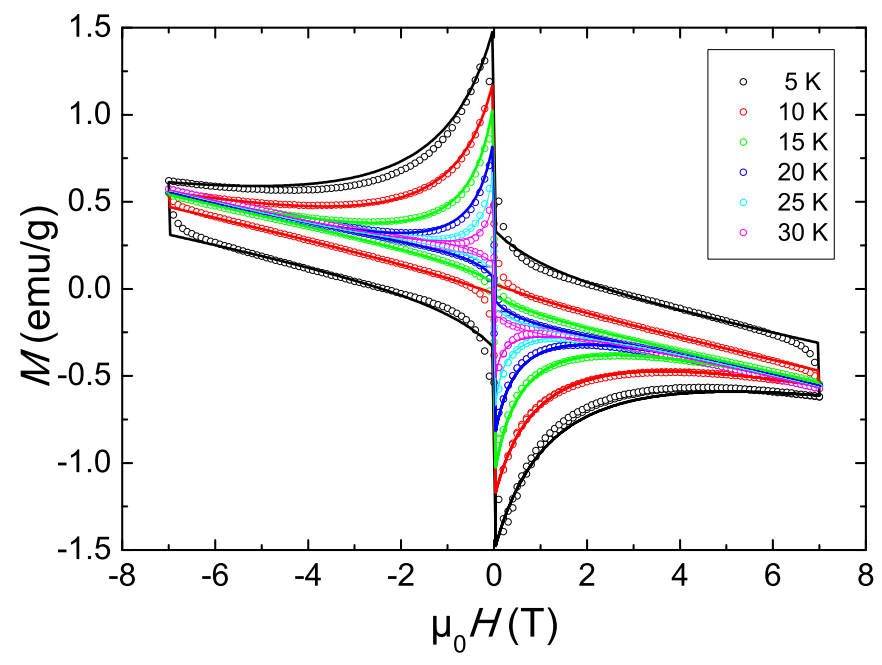

FIG. 2. $M(H)$ loops of the Bi-2212 nanowire network samples. The data points represent the experimental data, and the solid lines are computed curves.

\section{B. Magnetization data}

The superconducting transition data in field-cooled (FC) and zero-field cooled (ZFC) modes measured in an applied field of $1 \mathrm{mT}$ have already been reported in previous work [26]. An $M-T$ diagram is presented in Fig. S6 of the Supplemental Material [55]. The onset of superconductivity takes place at $\sim 76 \mathrm{~K}$, which is somewhat lower than the bulk $T_{c}$ of the Bi-2212 phase (85 K), which was also observed in Ref. [18]. Here, we have to note that the nanowire diameter is comparable to the London penetration depth, so we may attribute the reduced transition temperature to thermal and quantum fluctuations [27]. The onset of irreversibility is clearly visible further at $\sim 70 \mathrm{~K}$, where the FC and ZFC curves merge together.

Figure 2 shows magnetization hysteresis loops of the nanowire network sample in the temperature range of 5-30 K. At higher temperatures a hysteresis exists at low magnetic fields only. The hysteresis loops are asymmetric and look to be tilted in the clockwise direction. This will be analyzed in detail below. Hysteresis loops measured at elevated temperatures 40-70 K are presented in Fig. S8 of the Supplemental Material [55]. These loops were not considered for the fitting procedure.

Before introducing of the fitting detail, it is necessary to recall how the current flows through such a granular, polycrystalline sample. Here, we use the quantitative model established by Senoussi [21]. It demonstrates the relationship between the magnetic critical current $J_{\mathrm{mag}}$ and the lowfield magnetization structure of the polycrystalline materials. Furthermore, the model separates the current into two types of current loops: the high-field one related to the current inside single grains and the low-field one connected with the intergrain weak-link network. Figure 3 gives a schematic view of the current behavior inside a polycrystalline nanowire and the network. In Fig. 3(a), the bold circular arrows represent the current inside the individual grains while the dashed lines refer to the currents passing through the grains over several grain boundaries which are weak links showing a different magnetic field response, while in Fig. 3(b), the main current flows among the nanowires are shown by the red arrows. (a)

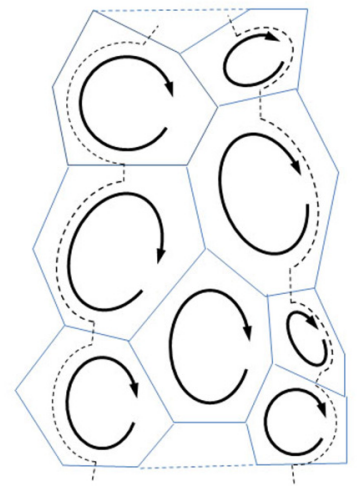

(b)

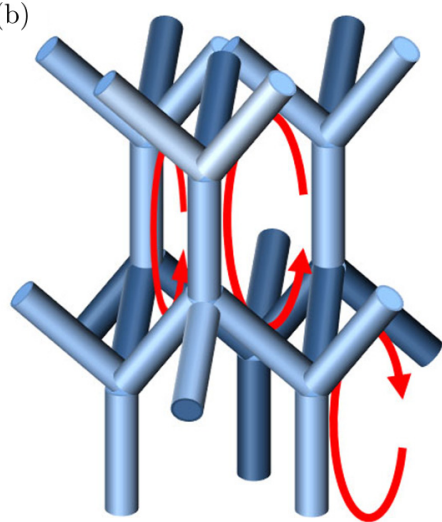

FIG. 3. (a) Model of current flow through an individual nanowire. The bold circular arrows represent the intragrain currents. The dashed lines indicate the current flow through different grain boundaries, which pose weak links to the currents. (b) The scheme of the nanowire network. The red arrows indicate the current flow between the individual nanowires.

Additionally, in the nanowire networks, there is another type of boundary formed by the interconnections of the nanowires (see Fig. 1(b) and Fig. S4 of the Supplemental Material [55]). These interconnections are formed during the heat treatment process, and their existence and field behavior are essential to the performance of the nanowire networks studied here.

\section{Fitting}

The measured $M(H)$ loops are considered as a superposition of a hysteresis loop $M_{S}(H)$ of the superconducting nanowires and an additional diamagnetic magnetization, $M_{D}(H)$. The additional diamagnetic signal is still present at high $H$, where contributions from both currents that cross between junctions and currents internal to the wires have disappeared. This signal is suggested to be due to diamagnetic $\mathrm{Bi}$ atoms. To extract the superconducting magnetization loops $M_{S}(H)$ the diamagnetic magnetization $M_{D}(H)=\chi_{m} H$ is to be subtracted from the experimental $M(H)$ loops. It is plausible to observe the high-field portion of $M(H)$ curves above $H_{c 2}$, where $M_{S}(H)=0$. We operated at smaller $H$ so the value of $\chi_{m}$ is selected to obtain $\left|d M_{S}^{+} / d H\right| \approx\left|d M_{S}^{-} / d H\right|$ at high $H$, where $M_{S}^{-(+)}$denotes the branch of the $M_{S}(H)$ loop during increase (decrease) of $H$. It is found that the obtained $M_{S}(H)$ loops are not tilted in high $H$. The observed asymmetry of the $M_{S}(H)$ loops relative to the $M=0$ axis is rather typical for high- $T_{c}$ superconductors.

The extended critical state model $[23,24]$ is relevant here due to a small size of the nanowires. The ECSM accounts for the equilibrium magnetization of the sample surface that results in the asymmetry of the $M(H)$ loop relative to the $M=0$ axis. The depth of the surface region with the equilibrium magnetization, $l_{s}$, is not higher than the magnetic field penetration depth $\lambda$ [28]. The magnetic field dependence of $l_{s}$ is defined by the superconductor class as for $\lambda(H)$. A quadratic dependence of $\lambda(H)$ is usual for conventional superconductors and a linearly increased dependence of $\lambda(H)$ was found in Bi-2212 superconductors [29]. In addition, 
TABLE I. Fitting parameters and estimated values of ECSM. The critical current density $j_{c 0}$ determines the width $(\Delta M)$ of the magnetization hysteresis. The depth of the surface layer with equilibrium magnetization $l_{s 0}$ determines the hysteresis asymmetry relative to $H$ axis. $H_{c 2}$ gives decreasing rate for $j_{c}(B)$, and $H_{\text {irr }}$ gives increasing rate for $l_{s}(H)$.

\begin{tabular}{lclcccc}
\hline \hline$T(\mathrm{~K})$ & $j_{c 0}\left(10^{6} \mathrm{~A} / \mathrm{cm}^{2}\right)$ & $l_{s 0} / R$ & $\mu_{0} H_{c 2}(\mathrm{~T})$ & $\mu_{0} H_{\mathrm{irr}}(\mathrm{T})$ & $J_{c 0}\left(10^{6} \mathrm{~A} / \mathrm{cm}^{2}\right)$ & $\mu_{0} H_{p}(\mathrm{mT})$ \\
\hline 5 & 16.9 & 0.15 & 85 & $\sim 80$ & 3.4 & 3.3 \\
10 & 13.7 & 0.19 & 70 & $35 \pm 8$ & 6.0 & 27.3 \\
15 & 12.0 & 0.205 & 42 & 13 & 4.8 & 23.8 \\
20 & 10.3 & 0.225 & 30 & 3.3 & 2.7 & 16.8 \\
25 & 8.75 & 0.25 & 23 & 2 & 2.5 & 13.0 \\
30 & 7.0 & 0.29 & 20 & & \\
\hline \hline
\end{tabular}

the dependence of $l_{s}(H)$ contains all the information of an averaged pinning landscape in the sample.

The magnetization is by definition $M(H)=-H+$ $\bar{B}(H) / \mu_{0}$, where $\bar{B}$ is the averaged magnetic field in the sample, and $\mu_{0}$ is the magnetic constant. The averaged magnetic field $\bar{B}$ results from integrating of the local magnetic field $B(r)$ inside the sample, and $r$ is the position vector. The local critical current density $j_{c}(B)$ and $B(r)$ are connected by Ampère's circuital law $d B / d r= \pm \mu_{0} j_{c}(B)$. The flux pinning provides $j_{c}(B) \sim 1 /|B|^{\alpha}$. Distortions of $j_{c}(B)$ and $l_{s}(H)$ by the peak effect can be accounted for [30].

The magnetic field penetration in the nanowire network is influenced by the porosity and the grain distribution [31]. The magnetic properties of the polycrystalline sample at high fields can be reduced to the characteristics of a single averaged grain. Then, the magnetization of superconducting phase is determined by $M_{S}(H)=-H+P_{S} \bar{B}(H) / \mu_{0}$, where $P_{S}$ is the volume content of superconducting phase in the sample. Taking the theoretical density of Bi-2212 $\left(6.4 \mathrm{~g} / \mathrm{cm}^{3}\right)$ [25] for the individual nanowires, we found the relative superconducting content $P_{S}$ to be $0.72 \%$ only.

To compute $M_{S}(H)$ loops one should use $j_{c}(B)$ and $l_{s}(H)$ dependencies satisfying the following requirements: the $j_{c}(H)$ dependence decreases from $j_{c 0}$ at $B=0$ to 0 at $B \geqslant B_{c 2}=$ $\mu_{0} H_{c 2}$; the $l_{s}(H)$ dependence increases from $l_{s 0}$ at $H=0$ to $R$ at $H \geqslant H_{\text {irr }}$ where the magnetization loops becomes reversible. $R$ is the radius of current circulation at high $H$. We express $j_{c}(B)$ and $l_{s}(H)$ as

$$
\begin{gathered}
j_{c}(B)=j_{c 0} \frac{1-\left|B / B_{c 2}\right|^{\alpha}}{1+\left|B /\left(h_{1} B_{c 2}\right)\right|^{\alpha}}, \\
l_{s}(H)=R-\left(R-l_{s 0}\right)\left(1-\mid H / H_{\mathrm{irr} \mid}\right)^{\beta},
\end{gathered}
$$

where $\alpha, \beta$, and $h_{1}$ are positive dimensionless coefficients, $\alpha, \beta \leqslant 1, h_{1} \ll 1$. Equation (1) is based on the Kim relation [32], which describes the $j_{c}(B)$ dependence due to the vortex pinning. As was known long ago, the Kim formula does not give overall agreement with experimental dependencies [33-35]. Urban modified the Kim relation to obtain $j_{c}(B)=0$ at $B=B_{c 2}$ [36]. This allows us to describe both low-field and high-field regions of $j_{c}(B)$ dependence. To improve the overall agreement, several versions of the Kim formula with a power-law behavior were applied in some works in the literature $[33,37,38]$. Equation (2) is a phenomenological fit and it qualitatively agrees with the $\lambda(H)$ dependence [29].
The $M_{S}(H)$ loops at different temperatures were computed for the case of a long cylindrical sample with radius $R$. The width of the loops is fitted by $j_{c 0} \times R$ and the asymmetry relative to the $M=0$ axis is fitted by $l_{s 0} / R$. The average nanowire radius of $\sim 160 \mathrm{~nm}$ obtained from SEM images[16] was used as the $R$ value. This value is somewhat smaller than the known $\lambda$ of Bi-2212 such that the high asymmetry of the $M_{S}(H)$ loops is not surprising. The fitting parameters at different temperatures are presented in Table I. All $M_{S}(H)$ curves are computed with $h_{1}=0.02, \alpha=0.63$. The parameter $\beta$ served for a fine tuning of fitting curves. $\beta$ equals 1 for most curves, but better agreement was reached at lower $T$ with $\beta=$ 0.4 for $5 \mathrm{~K}, \beta=0.8$ for $10 \mathrm{~K}$. Figure 4 demonstrates separate $M_{S}(H)$ and $M_{D}(H)$ curves for the magnetization loop at $T=$ $15 \mathrm{~K}$. The computed curves $M(H)=M_{S}(H)+M_{D}(H)$ are presented in Fig. 2 (solid lines).

Additionally, the penetration field $H_{p}$ was computed by using ECSM (see Table I). It should be noted that the computed $H_{p}$ values are slightly smaller than the product of $j_{c 0}$ and $R$, which equals the penetration field in the Bean model. The temperature dependencies of $H_{c 2}, H_{\text {irr }}$, and $H_{p}$ together with $j_{c 0}(T) R$ are plotted in Fig. 5. The temperature evolution of $H_{\text {irr }} / H_{c 2}$ is shown in the inset to Fig. 5. The temperature dependence of $H_{\text {irr }}$ of the present samples is similar to other Bi-2212 materials investigated in the literature.

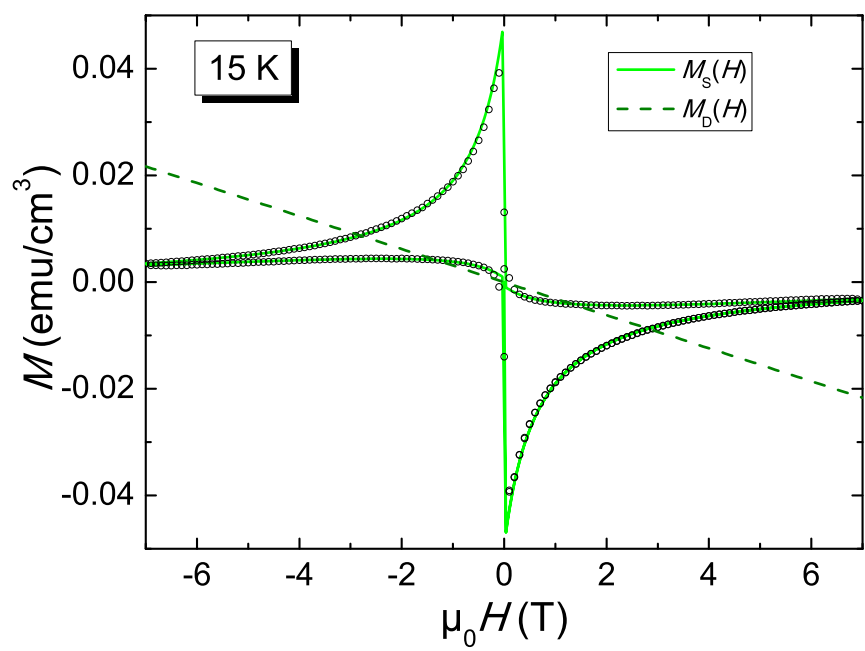

FIG. 4. Superconducting $M_{S}$ and diamagnetic $M_{D}$ magnetizations. The data points give the experimental loop tilted in the anticlockwise direction; the lines represent the computed curves. 


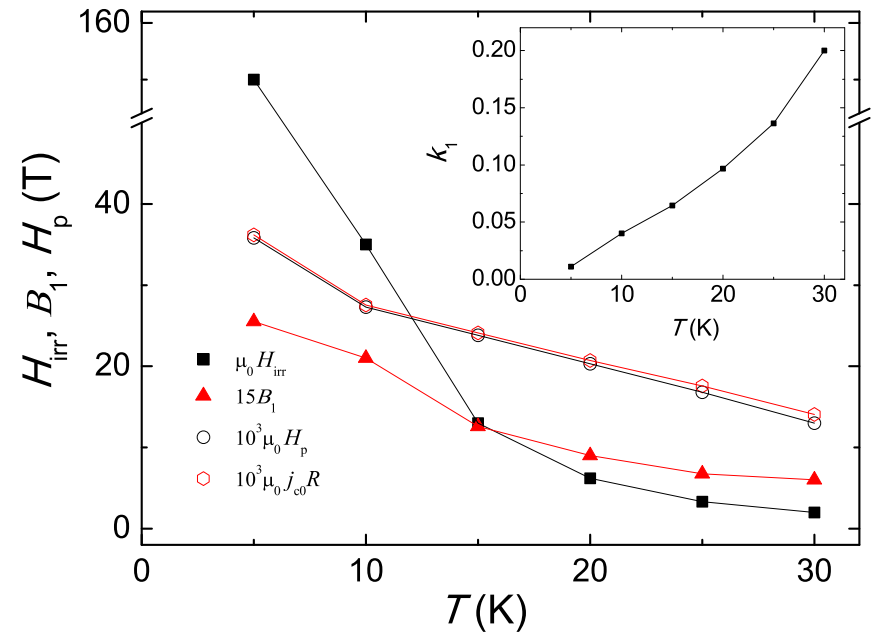

FIG. 5. The upper critical field $H_{c 2}$, the irreversibility field $H_{\text {irr }}$, and the penetration field $H_{p}$ at different temperatures. The inset displays the temperature dependence of $H_{\text {irr }} / H_{c 2}$. The lines are guides to the eye.

\section{Estimations of $J_{c}$ and $F_{p}$}

The nanowire surface region is useless for the supercurrent transport because Abrikosov vortices are not pinned there. Therefore the intragrain critical current density $J_{c}$ depends on $l_{s} / R$ :

$$
J_{c}(H) \approx j_{c}\left(\mu_{0} H\right)\left[1-l_{s}(H) / R\right]^{n},
$$

where $j_{c}\left(\mu_{0} H\right)$ is the local critical current density (1) near the nanowire surface, and $n$ is the index defined by the geometry of the grain, $n=3$ for a cylindrical sample and $n=2$ for a thin plate [39]. Expressing $J_{c}$ at $H=0$ as $J_{c 0}=j_{c 0}\left(1-l_{s 0} / R\right)^{n}$, we obtain the $J_{c}(H)$ dependence of ECSM:

$$
J_{c}(H)=J_{\mathrm{c} 0} \frac{1-\left|H / H_{c 2}\right|^{\alpha}}{1+\left|H /\left(h_{1} H_{c 2}\right)\right|^{\alpha}}\left(1-\left|H / H_{\mathrm{irr}}\right|\right)^{\gamma},
$$

where $\gamma=\beta \times n$. This $J_{c}(H)$ dependence decreases faster than $j_{c}(H)$, tending to 0 as $H$ approaches $H_{\text {irr }}(T)$. Formula (4) describes the $J_{c}(H)$ dependencies of different superconductors. The dependencies of $J_{c}(H)$ presented in Fig. 6 (lines) were calculated using $j_{c 0}$ and $l_{s 0} / R$ given in Table I and $n=3$. The calculated values of $J_{c 0}$ are given in Table I.

The intragrain critical current density was also estimated from the width of the magnetization loops $\Delta M$ by the Bean formula $J_{c}(H)=3 \Delta M /(2 R)$. The porosity is not accounted for in this formula such that resulting values should be increased $1 / P_{S}$ times for porous samples. The $J_{c}(H)$ dependencies resulting from the magnetization loops of Fig. 2 are presented in Fig. 6 (points). Given $R=160 \mathrm{~nm}$, the Bean model estimations are in good agreement with the computed data of ECSM.

The dependence of the pinning force density on the applied magnetic field was determined by $F_{p}(H)=\mu_{0} H \times J_{c}(H)$. In Fig. 7 , the normalized pinning force $f=F_{p} / F_{p 0}$ is plotted as a function of the reduced field $h=H / H_{\text {irr }}$ for different $T$; here $F_{p 0}$ is the maximal pinning force density at given $T$. The solid line in Fig. 7 represents the $f(h)$ dependence computed

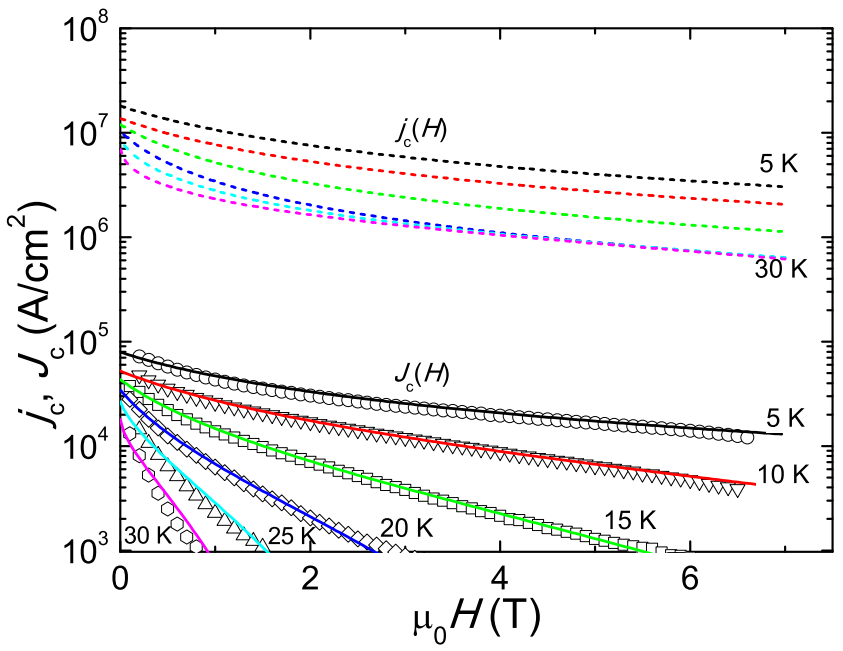

FIG. 6. The dependence of the intragrain critical current density on the magnetic field. The lines are ECSM curves. The points are the critical current density obtained from evaluating the width of the experimental loops (the Bean formula).

using Eq. (4) and the parameters from Table I. The Bean model estimations are plotted as points.

The resulting $f(h)$ dependencies at all temperatures are almost coinciding. The scaling law of Dew-Hughes (DH) [40] is used to fit the $f(h)$ dependence:

$$
\frac{F_{p}(H, T)}{F_{p 0}(T)}=\left(\frac{h}{h_{0}}\right)^{p}\left(\frac{1-h}{1-h_{0}}\right)^{q},
$$

where $h_{0}$ is the position of the maximum, $h_{0}=p /(p+q)$. The maximum flux pinning is found at $h_{0} \approx 0.11$ for all $T$. The scaled curves are fitted when $p=0.5$ and $q=4$ (dotted line in Fig. 7). These values differ from the Kramer parameters ( $p=0.5, q=2$ ), but they are typical for anisotropic and polycrystalline superconductors $[41,42]$.

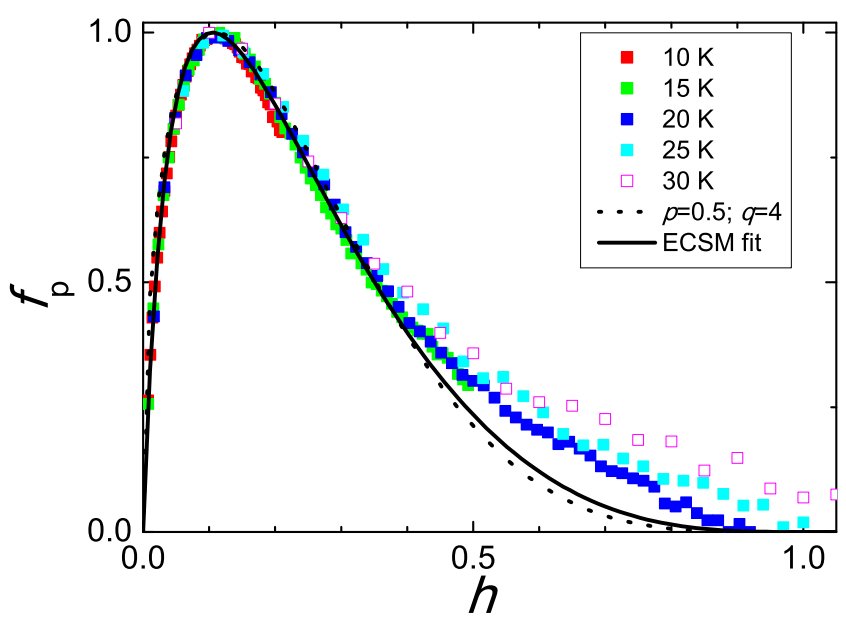

FIG. 7. Scaling of the pinning forces. The data points are obtained from the width of the experimental loops (the Bean formula). The solid line is the ECSM fitting curve for $T=15 \mathrm{~K}$; the dotted line gives the DH function with $p=0.5$ and $q=4$. 


\section{DISCUSSION}

The application of ECSM is especially relevant for polycrystalline superconductors in which the grain size is comparable with $\lambda$. Despite the incorporated nanowire radius being smaller than $\lambda$, the magnetization loops of the nonwoven superconducting fabrics are analogous to hysteresis loops measured on bulk Bi-2212 samples. This similarity calls for applying ECSM to the case of the nanowire network samples. All the estimated results from the model are shown in Table I. A successful fitting was reached even though the ECSM employed here disregards the disorder of the nanowires, the anisotropy, and the demagnetizing factor. The neglect of all of these can result in underestimations of the $j_{c}$ values, but does not affect the resulting $J_{c}$ values. The $j_{c 0}$ values of the Bi-2212 nanowires were estimated to decrease from $1.69 \times 10^{7} \mathrm{~A} / \mathrm{cm}^{2}$ to $7 \times 10^{6} \mathrm{~A} / \mathrm{cm}^{2}$ as $T$ increases from 5 to $30 \mathrm{~K}$.

At the same temperature range, the $J_{c 0}$ values of the ultraporous nonwoven fabric decrease from $10.4 \times 10^{6} \mathrm{~A} / \mathrm{cm}^{2}$ to $2.5 \times 10^{6} \mathrm{~A} / \mathrm{cm}^{2}$. Many experiments with Bi-2212 whiskers [43] give the intragrain critical current density similar to values in the sixth column of Table I. At the same time these values are a few orders of magnitude greater than the transport critical current density of Bi-2212 wires [44] that is strongly limited by weak-linked grain boundaries.

It is seen that Eq. (4) of the ECSM model gives surprisingly good agreement with the $J_{c}(H)$ dependencies as determined from the $M(H)$ loops by the Bean model (Fig. 6) as well as the field dependencies of the pinning force (Fig. 7). It is important to note that the $J_{c}(H)$ values estimated using the Bean model and ECSM are nearly coinciding only when the same value of $R$ is used in both models and the porosity is accounted for. As it was tested for many different computed magnetization loops, the good coincidence keeps for for any values of $R$. The reason for this quantitative coincidence is the same influence of the surface on the macroscopic critical current and on the irreversible magnetization $\Delta M$. The Bean model establishes the relation between the irreversible magnetization $\Delta M(H)$ and the $J_{c}(H)$ dependence. The $J_{c}(H)$ dependence of a smaller sample decreases faster than $J_{c}(H)$ of a larger sample. This is due to omitting the surface region with the field-dependent depth $l_{s}(H)$. The irreversible magnetization of smaller samples also decreases faster than $\Delta M(H)$ of large samples. As the numerical calculations display, the dependencies $J_{c}(H)$ and $\Delta M(H)$ decrease identically and both equal 0 at $H$ higher than the irreversibility field. The exact consideration of the magnetization of porous, polycrystalline superconductors could be much more complicated. So, an equivalent superconducting sample, whose radius equals the average grain radius, is considered instead. With this simplification, the Bean model can be used to estimate the $J_{c}$ values of porous, polycrystalline superconductors. It should be noted here that long before now many researches began to apply the Bean formula to estimate $J_{c}$ of polycrystalline superconductors from magnetic measurements (see, e.g., Ref. [45]).

The macroscopic intergrain current contributes negligibly to magnetization loops of ultraporous polycrystalline superconductors. We support that the macroscopic current induces a small contribution to the magnetization detected at low $H<H_{p}$, where the experimental data points are fitted worse by ECSM accounting for the intragrain critical current only.
This intergrain contribution is about $\sim J_{c \text {,tr }} R_{s}$, where $J_{c \text {,tr }}$ is the transport critical current density and $R_{s}$ is the sample radius. A rough estimation of $J_{c \text {,tr }}$ is obtained from $J_{c \text {,tr }} R_{s} \ll J_{c} R P_{S}$; the right part is the intragrain contribution to the magnetization. Given $J_{c}=10^{7} \mathrm{~A} \mathrm{~cm}^{-2}, R=160 \mathrm{~nm}, R_{s}=2.1 \mathrm{~cm}$, and $P_{S}=$ 0.00072 , we obtain $J_{c \text {,tr }} \ll 0.055 \mathrm{~A} \mathrm{~cm}^{-2}$ at $T=5 \mathrm{~K}$. Similar low values of the transport critical current density in the Bi2212 nanowire network were observed in previous work [19].

The penetration field decreases with increasing $T$ as well as $j_{c 0}(T)$. The low values of $H_{p}(<40 \mathrm{mT})$ are due to the small size of the nanowires. The estimated temperature dependence of $H_{c 2}$ shows a positive curvature (see Fig. 5), which is similar to the $H_{c 2}(T)$ dependency of many HTSc $[46,47]$. The temperature dependence of $H_{\text {irr }} / H_{c 2}$ is qualitatively described by the equations given by Fabbricatore et al. [41]. An accurate calculation of $H_{c 2}(T) / H_{\text {irr }}(T)$ requires more information about the $H_{c 2}(T)$ and the coherence length $\xi(T)$ dependencies.

The irreversibility field, $H_{\text {irr }}$, of the nanowire network sample and its temperature dependence are comparable to those of filaments extracted from $\mathrm{Bi}-2212 / \mathrm{Bi}-2223$ tape samples $[48,49]$, even though the grains within an individual nanowire do not exhibit a texture as seen from the TEM images. This indicates a proper formation of the $\mathrm{Bi}-2212$ phase within our nanowire samples.

The pinning force scaling reveals a peak position, $h_{0}$, of 0.11 , which is smaller than the typical result for $\mathrm{Bi}-2212$ samples of $h_{0}=0.2$ (denoting flux pinning at 2D objects like grain boundaries). Following the analysis of Eisterer [42], this reflects the influence of anisotropy and percolation. The nanowires are polycrystalline without a specific texture of the grains, so the supercurrents flowing within a nanowire have to pass grains with many different orientations, and the numerous interconnections between the nanowires form a percolation network. An anisotropy larger than 4 yields a position of $h_{0}=0.1$ for the grain boundary pinning, which is certainly fulfilled for $\mathrm{Bi}-2212$. In contrast, the influence of percolation effects on the peak position is much smaller [42].

One should note that the magnetic measurements on these nanowire arrays give important information about the intragrain current density, the irreversibility fields, and the flux pinning behavior. These data can be readily compared to data of other superconducting materials. In contrast, conclusions about the intergrain currents and their properties are only possible when modeling the nanowire networks, as there are variations in the nanowire diameter and in the pore size. Furthermore, the effective number of the interconnections between the individual nanowires is unknown, and also the physical properties of these interconnections are not known. Due to this reason, electric transport measurements can only deliver a critical current $I_{c}$, but not a current density, $j_{c}$, without invoking modeling of the nanowire networks. To further elucidate the properties of the interconnections, further measurements will be required.

As the results obtained concerning the current densities and the magnetic field behavior are quite encouraging, one may think of possible application areas for this new class of superconducting materials. Here, several directions are possible. Individual nanowires may see applications as sensor elements like their magnetic counterparts of $(\mathrm{La}, \mathrm{Sr}) \mathrm{MnO}_{3}$ [50]. The as-spun nanowire arrays could be dissolved again in a suitable 
agent to enable the use of the material as a ink for ink-jet printing [51]. For the entire nanowire networks, the extremely low weight and the possible large size are the essential points. This could enable applications as fault current limiters, and, e.g., for applications in space, so one may consider them as ultralight shielding layers [52], eventually reinforced together with suitable polymers [53] as large-scale superconducting mats or as building blocks for nanoporous, bulk superconductors [54]. The reinforcing with suitable polymers is an essential means to overcome the brittleness of the as-prepared nanowire network samples. Overall, several applications of these nanowire networks are to be tested in the future.

\section{CONCLUSIONS}

Bi-2212 nonwoven nanowire networks were fabricated using the electrospinning technique. The samples were characterized in detail by SQUID magnetometry. The extended critical state model was applied to analyze the magnetic properties of the nanowire network superconductors, which are characterized by an extremely low density and low weight. Accounting for the sample porosity and the averaged nanowire diameter, we find connection between the critical current density of an averaged nanowire and the one of the nanowire network. The critical current densities and the irreversibility fields of the nanowires in the samples have similar values and follow the same laws as bulk Bi-2212 samples. The high porosity of the samples results in much lower values of the critical current density of the nonwoven nanowire networks than the ones of a single nanowire.

\section{ACKNOWLEDGMENTS}

We thank Prof. V. Presser (INM Saarbrücken and Saarland University) for the opportunity to use the electrospinning apparatus, and J. Schmauch (Saarland University, Prof. Birringer's group) for technical assistance. The collaboration UdS-Nancy was supported by the EU-INTERREG IVa, project "Greater Region Magnetism Network (GRMN)". This work was supported by the Volkswagen Foundation.
[1] D. C. Larbalestier, A. Gurevich, M. Feldmann, and A. Polyanskii, High- $T_{c}$ superconducting materials for electric power applications, Nature (London) 414, 368 (2001).

[2] R. M. Scanlan, A. P. Malozemoff, and D. C. Larbalestier, Superconducting materials for large-scale applications, Proc. IEEE 92, 1639 (2004).

[3] S. R. Foltyn, L. Civale, J. L. MacManus-Driscoll, Q. X. Jia, B. Maiorov, H. Wang, and M. Maley, Materials science challenges for high-temperature superconducting wire, Nat. Mater. 6, 631 (2007).

[4] E. Sudhakar Reddy and T. Rajasekharan, Fabrication of textured $\mathrm{REBa}_{2} \mathrm{Cu}_{3} \mathrm{O}_{7} / \mathrm{RE}_{2} \mathrm{BaCuO}_{5}(\mathrm{RE}=\mathrm{Y}, \mathrm{Gd})$ composites by infiltration and growth of $\mathrm{RE}_{2} \mathrm{BaCuO}_{5}$ preforms by liquid phases, Supercond. Sci. Technol. 11, 523 (1998).

[5] P. Missak Swarup Raju, V. Seshubai, and T. Rajasekharan, A generic process to introduce nanoparticles into powder preforms and its application to infiltration growth processing of $\mathrm{REBa}_{2} \mathrm{Cu}_{3} \mathrm{O}_{7}$ superconductor, Mater. Chem. Phys. 161, 59 (2015).

[6] K. Nakazato, M. Muralidhar, M. R. Koblischka, and M. Murakami, Fabrication of bulk Y-Ba-Cu-O superconductors with high critical current densities through the infiltration growth process, Cryogenics 63, 129 (2014).

[7] E. S. Reddy, J. G. Noudem, M. Tarka, and G. J. Schmitz, Mono-domain $\mathrm{YBa}_{2} \mathrm{Cu}_{3} \mathrm{O}_{y}$ superconductor fabrics prepared by an infiltration process, Supercond. Sci. Technol. 13, 716 (2000).

[8] E. S. Reddy and G. J. Schmitz, Recent developments in processing and properties of large grain superconducting YBCO fabrics, Supercond. Sci. Technol. 15, 727 (2002).

[9] Y. Shiohara and A. Endo, Crystal growth of bulk high- $T_{c}$ superconducting oxide materials, Mater. Sci. Eng. R 19, 1 (1997).

[10] J. G. Noudem, E. S. Reddy, M. Tarka, E. A. Goodilin, M. Noe, M. Zeisberger, and G. J. Schmitz, Electrical performance of single domain $\mathrm{YBa}_{2} \mathrm{Cu}_{3} \mathrm{O}_{y}$ fabrics, Physica $\mathrm{C}$ 366, 93 (2002).
[11] H. Wu, W. Pan, D. Lin, and H. Li, Electrospinning of ceramic nanofibers: Fabrication, assembly and applications, J. Adv. Ceramics 1, 2 (2012).

[12] M. Law, J. Goldberger, and P. D. Yang, Semiconductor nanowires and nanotubes, Annu. Rev. Mater. Res. 34, 83 (2004).

[13] D. Li, J. T. McCann, and Y. N. Xia, Electrospinning: A simple and versatile technique for producing ceramic nanofibers and nanotubes, J. Am. Ceram. Soc. 89, 1861 (2006).

[14] J. M. Li, X. L. Zeng, A. D. Mo, and Z. A. Xu, Fabrication of cuprate superconducting $\mathrm{La}_{1.85} \mathrm{Sr}_{0.15} \mathrm{CuO}_{4}$ nanofibers by electrospinning and subsequent calcination in oxygen, CrystEngComm 13, 6964 (2011).

[15] X. L. Zeng, M. R. Koblischka, and U. Hartmann, Synthesis and characterization of electrospun superconducting $(\mathrm{La}, \mathrm{Sr}) \mathrm{CuO}_{4}$ nanowires and nanoribbons, Mater. Res. Express 2, 095022 (2015).

[16] X. L. Zeng, M. R. Koblischka, T. Karwoth, T. Hauet, and U. Hartmann, Preparation of granular Bi-2212 nanowires by electrospinning, Supercond. Sci. Technol. 30, 035014 (2017).

[17] E. A. Duarte, N. G. Rudawski, P. A. Quintero, M. W. Meisel, and J. C. Nino, Electrospinning of superconducting YBCO nanowires, Supercond. Sci. Technol. 28, 015006 (2015).

[18] E. A. Duarte, P. A. Quintero, M. W. Meisel, and J. C. Nino, Electrospinning synthesis of superconducting BSCCO nanowires, Physica C 495, 109 (2013).

[19] M. R. Koblischka, X. L. Zeng, T. Karwoth, T. Hauet, and U. Hartmann, Transport and magnetic measurements on Bi-2212 nanowire networks prepared via electrospinning, IEEE Trans. Appl. Supercond. 26, 1800605 (2016).

[20] C. P. Bean, Magnetization of Hard Superconductors, Phys. Rev. Lett. 8, 250 (1962).

[21] S. Senoussi, Review of the critical current densities and magnetic irreversibilities in high- $T_{c}$ superconductors, J. Phys. III France 2, 1041 (1992).

[22] D. X. Chen, R. W. Cross, and A. Sanchez, Effects of critical current density, equilibrium magnetization and surface barrier on 
magnetization of high temperature superconductors, Cryogenics 33, 695 (1993).

[23] D. M. Gokhfeld, D. A. Balaev, M. I. Petrov, S. I. Popkov, K. A. Shaykhutdinov, and V. V. Val'kov, Magnetization asymmetry of type-II superconductors in high magnetic fields, J. Appl. Phys. 109, 033904 (2011).

[24] D. M. Gokhfeld, An extended critical state model: Asymmetric magnetization loops and field dependence of the critical current of superconductors, Phys. Solid State 56, 2380 (2014).

[25] P. N. Mikheenko, K. K. Uprety, and S. X. Dou, BSCCO, in Handbook of Superconducting Materials, Vol. I, edited by D. A. Cardwell and D. S. Ginley (IOP Publishing, Bristol, UK, 2003).

[26] M. R. Koblischka, X. L. Zeng, T. Karwoth, T. Hauet, and U. Hartmann, Magnetic properties of electrospun non-woven superconducting fabrics, AIP Adv. 6, 035115 (2016).

[27] D. S. Golubev and A. D. Zaikin, Thermally activated phase slips in superconducting nanowires, Phys. Rev. B 78, 144502 (2008).

[28] L. Burlachkov, Magnetic relaxation over the Bean-Livingstone surface barrier, Phys. Rev. B 47, 8056 (1993).

[29] A. Maeda, Y. Iino, T. Hanaguri, N. Motohira, K. Kishio, and T. Fukase, Magnetic-Field Dependence of the London Penetration Depth of $\mathrm{Bi}_{2} \mathrm{Sr}_{2} \mathrm{CaCu}_{2} \mathrm{O}_{y}$, Phys. Rev. Lett. 74, 1202 (1995).

[30] D. M. Gokhfeld, Secondary peak on asymmetric magnetization loop of type-II superconductors, J. Supercond. Novel Magn. 26, 281 (2013).

[31] D. M. Gokhfeld, D. A. Balaev, S. I. Popkov, K. A. Shaykhutdinov, and M. I. Petrov, Magnetization loop and critical current of porous Bi-based HTS, Physica C 434, 135 (2006).

[32] Y. B. Kim, C. F. Hempstead, and A. R. Strnad, Critical Persistent Currents in Hard Superconductors, Phys. Rev. Lett. 9, 306 (1962).

[33] F. Irie and K. Yamafuji, Theory of flux motion in non-ideal type-II superconductors, J. Phys. Soc. Jpn. 23, 255 (1967).

[34] G. R. Kumar and P. Chaddah, Extension of Bean's model for high- $T_{c}$ superconductors, Phys. Rev. B 39, 4704 (1989).

[35] V. V. Val'kov and B. P. Khrustalev, Magnetization of granular high- $T_{c}$ superconductors in strong magnetic fields, JETP 80, 680 (1995).

[36] E. W. Urban, Flux flow and a new critical-current formula, J. Appl. Phys. 42, 115 (1971).

[37] J. N. Jones, P. R. Wilshaw, D. Dew-Hughes, and K. A. Johnson, The low field simulation and study of magnetization in high$T_{c}$ superconductors at $77 \mathrm{~K}$, Supercond. Sci. Technol. 5, S351 (1992).

[38] M. Zehetmayer, Simulation of the current dynamics in superconductors: Application to magnetometry measurements, Phys. Rev. B 80, 104512 (2009).

[39] D. M. Gokhfeld, Critical current density and trapped field in HTS with asymmetric magnetization loops, J. Phys.: Conf. Ser. 695, 012008 (2016).

[40] D. Dew-Hughes, Flux pinning mechanisms in type-II superconductors, Philos. Mag. 30, 293 (1974).
[41] P. Fabbricatore, C. Priano, A. Sciutti, G. Gemme, R. Musenich, R. Parodi, F. Gomory, and J. R. Thompson, Flux pinning in Bi-2212/Ag-based wires and coils, Phys. Rev. B 54, 12543 (1996).

[42] M. Eisterer, Calculation of the volume pinning force in $\mathrm{MgB}_{2}$ superconductors, Phys. Rev. B 77, 144524 (2008).

[43] P. Badica, K. Togano, S. Awaji, K. Watanabe, and H. Kumakura, Review on Bi-Sr-Ca-Cu-O whiskers, Supercond. Sci. Technol. 19, R81 (2006).

[44] F. Kametani, J. Jiang, M. Matras, D. Abraimov, E. E. Hellstrom, and D. C. Larbalestier, Comparison of growth texture in round $\mathrm{Bi}_{2} \mathrm{Sr}_{2} \mathrm{CaCu}_{2} \mathrm{O}_{8}$ and flat $\mathrm{Bi}_{2} \mathrm{Sr}_{2} \mathrm{Ca}_{2} \mathrm{Cu}_{3} \mathrm{O}_{10}$ wires at its relation to high critical current density development, Sci. Rep. 5, 8285 (2015).

[45] D. X. Chen and R. B. Goldfarb, Kim model for magnetization of type-II superconductors, J. Appl. Phys. 66, 2489 (1989).

[46] M. S. Osofsky, R. J. Soulen, Jr., S. A. Wolf, J. M. Broto, H. Rakoto, J. C. Ousset, G. Coffe, S. Askenazy, P. Pari, I. Bozovic, J. N. Eckstein, and G. F. Virshup, Anomalous Temperature Dependence of the Upper Critical Magnetic Field in Bi-Sr-Cu-O, Phys. Rev. Lett. 71, 2315 (1993).

[47] Y. Ando, G. S. Boebinger, A. Passner, L. F. Schneemeyer, T. Kimura, M. Okuya, S. Watauchi, J. Shimoyama, K. Kishio, K. Tamasaku, N. Ichikawa, and S. Uchida, Resistive upper critical fields and irreversibility lines of optimally doped high- $T_{c}$ cuprates, Phys. Rev. B 60, 12475 (1999).

[48] M. R. Koblischka and J. Sosnowski, Temperature-dependent scaling of pinning force data in Bi-based high- $T_{c}$ superconductors, Eur. Phys. J. B 44, 277 (2005).

[49] I. Chong, Z. Hiroi, and M. Izumi, High critical-current density in the heavily $\mathrm{Pb}$-doped $\mathrm{Bi}_{2} \mathrm{Sr}_{2} \mathrm{CaCu}_{2} \mathrm{O}_{8+\delta}$ superconductor: Generation of efficient pinning centers, Science 276, 770 (1997).

[50] D. Xu, L. Luo, Y. Ding, and P. Xu, Sensitive electrochemical detection of glucose based on electrospun $\mathrm{La}_{0.88} \mathrm{Sr}_{0.12} \mathrm{MnO}_{3}$ nonofibers modified electrode, Anal. Biochem. 489, 38 (2015).

[51] M. Vilardell, X. Granados, S. Ricart, I. Van Driessche, A. Palau, T. Puig, and X. Obradors, Flexible manufacturing of functional ceramics coatings by inkjet printing, Thin Solid Films 548, 489 (2013).

[52] S. G. Haupt, G. R. Riley, and J. T. McDevitt, Conductive polymer/high-temperature superconductor composite structures, Adv. Mater. 5, 755 (1993).

[53] S. Posen, M. K. Transtrum, G. Catelani, M. U. Liepe, and J. P. Sethna, Shielding Superconductors with Thin Films as Applied to rf Cavities for Particle Accelerators, Phys. Rev. Appl. 4, 044019 (2015).

[54] P. Fiertek, B. Andrzejewski, and W. Sadowski, Synthesis and transport properties of porous superconducting ceramics of $\mathrm{YBa}_{2} \mathrm{Cu}_{3} \mathrm{O}_{7-\delta}$, Rev. Adv. Mater. Sci. 23, 52 (2010).

[55] See Supplemental Material at http://link.aps.org/supplemental/ 10.1103/PhysRevMaterials.1.044802 for more information. 